\title{
L'héritage sexualisé de l'esclavage et la formation de l'identité dans Pension les Alizés et Histoire de la femme cannibale
}

https://doi.org/10.29173/af29435

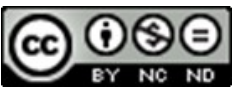

Caitlin Midgley

caitlin.midgley@alumni.ubc.ca

University of British Columbia, Canada

Résumé. Les femmes de la diaspora africaine ont été marquées par l'héritage traumatique sexualisée de l'esclavage; un héritage qui affecte leur capacité à établir des relations et des identités. Dans le contexte de cet héritage, Condé explore dans deux genres différents l'impact du traumatisme et du racisme sur la capacité de deux femmes de la diaspora africaine à établir des identités et à trouver l'amour. Dans la première œuvre, une pièce de théâtre, Pension les Alizés, le protagoniste une danseuse exotique à Paris, ne peut pas se débarrasser de la tache de l'esclavage ou établir des relations durables et dans la vieillesse elle se perd dans des illusions; elle est toujours accrochée à son identité de danseuse d'autrefois. Dans une œuvre ultérieure, Histoire de la femme cannibale, un roman, Condé est plus optimiste, le protagoniste parvient à se séparer de l'héritage de l'esclavage; elle commence à se forger une nouvelle identité en s'identifiant à la force de la femme cannibale. Les deux genres différents présentent différentes possibilités d'explorer l'héritage de l'esclavage. Compte tenu de la séparation de deux décennies, nous voyons l'évolution de la réflexion de Condé sur les réactions possibles au traumatisme historique collectif de l'esclavage.

Mots clés : Maryse Condé; la Traite; le traumatisme; l'identité; l'amour

Abstract. Women in the African diaspora have been scarred by the traumatic sexualized heritage of slavery; an inheritance that affects their ability to build relationships and identities. In the context of this heritage Conde explores in two different media the impact of trauma and racism on the ability of two different women of the African diaspora to find love and develop identities. In the first work, a play 
Pension les Alizés, the protagonist an exotic dancer in Paris, cannot rid herself of the stain of slavery or establish lasting relationships and in old age lives lost in illusions clinging to her identity as a once highly desired dancer. In a subsequent work, a novel Histoire de la femme cannibale Condé is more hopeful, the protagonist is able to separate herself from the legacy of slavery; she begins to forge a new identity as she identifies with a time before slavery. Given the two decade separation we see the evolution of Conde's thinking about possible responses to the collective historical trauma of slavery.

Keywords: Maryse Condé; slave trade; trauma; identity; love

$\mathrm{D}$ ans ses écrits Maryse Condé revient, à plusieurs reprises, sur les thèmes de l'aliénation raciale, de la lutte des femmes noires pour l'autonomie et de la quête identitaire dans le contexte de l'héritage de l'esclavage; un héritage que ses héroïnes ont souvent intériorisé. Ces mêmes thèmes se retrouvent dans sa pièce à deux, Pension les Alizés, publiée en 1988 et son roman Histoire de la femme cannibale, publié en 2005. Le roman a permis à Condé de revisiter les mêmes thèmes avec une description plus complète de la trame de fond, des intrigues plus complexes et un meilleur accès aux pensées intérieures des personnages. Les protagonistes sont deux femmes noires guadeloupéennes de la diaspora africaine, Emma et Rosélie. Dans Pension les Alizés, Emma est une Guadeloupéenne vieillissante qui, après une tentative infructueuse de devenir médecin, se fait danseuse exotique à Paris. Elle tombe amoureuse d'Ismaël, un docteur fuyant la dictature du président haïtien Jean-Claude Duvalier. Dans Histoire de la femme cannibale, Rosélie vit selon les caprices des hommes de sa vie. Ayant abandonné son intention d'étudier le droit à Paris, elle se retrouve prostituée dans un pays africain jusqu'à ce que Stephen, un professeur universitaire blanc vienne « à son secours ». Emma et Rosélie partagent des traumatismes personnels et l'héritage de l'esclavage ainsi qu'une quête d'amour et d'identité. De quelle manière les femmes protagonistes de Condé dans ces deux œuvres différentes réagissent-elles aux effets des traumatismes personnels et collectifs alors qu'elles tentent d'établir une identité et de former une relation amoureuse? Dans le contexte de l'héritage sexualisé de la traite négrière, nous allons d'abord examiner la notion de traumatisme, puis d'amour et enfin d'identité particulièrement en ce qui concerne les protagonistes dans Pension les Alizés et Histoire de la femme cannibale.

\section{LE TRAUMATISME}

L'un des héritages de la traite négrière est la mémoire collective traumatisante partagée par les descendants des premières victimes. La mémoire collective est la mémoire d'un groupe transmise d'une génération à l'autre. Selon Maurice Halbwachs, la mémoire collective se conserve « dans des groupes où nous sommes libres de pénétrer quand nous le voulons, dans des pensées collectives avec lesquelles nous restons toujours en rapport étroit » (Halbwachs 93). La mémoire collective n'est pas d'une importance égale pour tous les membres du groupe. Pour certains, c'est l'un des principaux déterminants de leur identité; pour d'autres, ce n'est qu'un aspect parmi d'autres. Emma dans Pension les Alizés et Rosélie dans Histoire de la femme cannibale sont profondément touchées par les conséquences historiques de l'esclavage, mais elles ont tendance à sous-estimer son importance. Comme le souligne Emily Sahakian : " Condé's play does not conspicuously address slavery, but slavery's sexualized legacy permeates the behaviours and attitudes of the Guadeloupean protagonist Emma and her Haitian lover Ishmael. » (Sahakian 397). De la même manière, le roman de Condé Histoire de la femme cannibale n'aborde pas directement l'esclavage, mais malgré les efforts de Rosélie pour s'en éloigner, elle se heurte à son héritage. La mémoire collective n'est pas nécessairement traumatique, mais dans le cas de la diaspora 
africaine, ce problème est difficile à éviter. Pour beaucoup, la plaie psychique de la Traite est toujours ouverte; cette plaie reflète un héritage de traumatisme collectif qui résonne à travers les siècles.

Selon la théorie des traumatismes, par nature, le traumatisme est à la fois soudain et durable; c'est l'impact psychique qui est si dévastateur qu'on ne peut y faire face dans l'immédiat. Caruth l'explique comme suit : " the trauma is experienced too early, too unexpectedly, to be fully known and is therefore not accessible to the conscience until it is imposed again and again, in the nightmares and repetitive actions of the survivor » (4). Le traumatisme peut être soit personnel, soit collectif. Dans les deux cas, le traumatisme est un événement qui se produit une fois, mais dont les effets sont durables, même transmis de génération en génération. Les plaies peuvent guérir, mais elles sont souvent rouvertes. Pour Caruth, la victime du traumatisme peut retrouver sa voix lorsque la plaie est réimposée : «A voice paradoxically released by the wound » (2). Dans le dernier chapitre de son livre Les Damnés de la terre, Frantz Fanon analyse « des plaies multiples et indélébiles » laissées par la colonisation, cette « pourvoyeuse des hôpitaux psychiatriques » (177). Dans Pension les Alizés et Histoire de la femme cannibale Emma et Rosélie sont toutes deux confrontées au traumatisme qui affecte leur sentiment de soi et leur capacité à établir des relations authentiques. Condé donne la parole à leurs plaies personnelles et collectives.

Dans Pension les Alizés, une grande partie des traumatismes vécus par Emma et son amant Ismaël sont liés au traumatisme collectif de la Traite négrière. Le traumatisme de l'esclavage imprègne la mémoire collective de la diaspora africaine et cette mémoire est partagée dans de nombreux pays. La similitude entre la Guadeloupe et Haïti est soulignée lorsqu' Ismaël s'interroge : « La Guadeloupe, Haïti, est-ce que ce n'est pas pareil! Est-ce que nous ne sommes pas le même peuple sorti du même ventre de négriers? Est-ce que nos créoles ne se ressemblent pas? Et nos proverbes et nos contes? Toi, tu dis Zamba, moi, je dis Bouki. Mais est-ce que ce n'est pas le même animal? » (Pension 99) et puis « Haïti, Martinique, Guadeloupe et Barbade et Jamaïque, est-ce que tout cela n'est pas pareil? Des cannes à sucre, des exilés par milliers, le poids de l'oppression! » (Pension 103). Condé souligne ici l'aspect commun des îles des Caraïbes, de leur histoire et de leur culture. S'il peut y avoir des différences entre les langues et les contes, ils ont une mémoire collective partagée. Dans un autre rappel historique d'époque de plantations, Emma se souvient du train de canne à sucre qui traversait la ville, malgré la fin de l'esclavage : « Il y avait un train de canne à sucre qui marchait en somnambule sur ses rails et nous les enfants nous courions après lui et nous nous suspendions aux wagons! En général, les filles n'étaient pas assez fortes. Mais, moi je tenais bon. » (Pension 54). Le train de canne à sucre évoque l'image de la puissance irréfléchie de l'exploitation des Noirs par les Blancs alors qu'il se déplace sans relâche vers sa destination. Qu'Emma se suspendait au wagon suggère tant sa force morale que physique. Ismaël reconnaît leur histoire commune comme un traumatisme collectif qui les a endommagés, mais aussi toute la diaspora africaine : « Mais j'ai surtout voulu discuter, comprendre pourquoi notre histoire est une suite d'erreurs, de projets avortés, de débarquement manqués. Qu'est-ce qui est mort en nous? D'Afrique aux Amériques, à genoux... » (Pension 70). Pour Ismaël, l'échec du complot contre Baby Doc, le dictateur brutal d'Haïti, reflète un traumatisme intérieur partagé; pour lui, l'échec était dû à une insuffisance qu'ils partageaient tous. Il voit l'échec non pas comme un événement unique, mais comme une répétition de leur traumatisme. Clorinde, l'amant d'Ismaël en Haïti, résume l'héritage de l'esclavage en six mots : «Pas de bonheur pour le nègre! » (Pension 72). Condé présente ici une critique sociale dévastatrice de l'héritage de l'esclavage; ce manque de bonheur reflète l'impact continu du traumatisme sur la diaspora africaine.

Alternative francophone

https://journals.library.ualberta.ca/af/index.php/af 
Ce traumatisme est collectif comme l'explique Emma : «Tous les enfants antillais ont peur de la nuit » (Pension 32). La lutte pour comprendre le traumatisme collectif ressurgit à l'âge adulte, ce qui correspond à la théorie du traumatisme de Caruth pour qui le traumatisme n'est pas entièrement compris tant qu'il ne s'est pas réimposé. Dans Pension les Alizés, le traumatisme est réimposé à travers les cauchemars d'Ismaël et d'Emma. Chaque fois qu'Ismaël s'endort, il souffre de cauchemars. Emma a aussi des cauchemars comme elle revit ce qu'elle perçoit comme son abandon de part de sa mère chaque nuit quand elle était enfant, sa mère la laissait seule dans le noir et dans sa peur (Pension 84). Les cauchemars qui lui reviennent tourmentent son âme, mais elle trouve du réconfort dans la musique. Pour Emma, la musique est un baume pour son âme, au moins temporairement : "Toutes les douleurs disparaissent. Les nouvelles et les très anciennes... » (Pension 44). Les très anciennes reflètent le traumatisme partagé par toutes les victimes de l'esclavage, traumatisme qui entrave l'établissement de relations intimes.

Le choix d'Emma de devenir danseuse exotique est au cœur de la pièce puisqu'elle se plie ainsi aux désirs de l'homme blanc. Elle ressent le besoin de justifier sa décision à Ismaël et à elle-même : «Papa s'était trompé sur mon compte. Je n'avais rien pour faire un médecin. D'abord j'ai peur du sang. Non, je ne supporte pas de voir le sang! Et puis, je ne suis pas très intelligente. Mon cerveau, il ne pèse pas lourd. Je n'avais qu'un corps. Je m'en suis servi. » (Pension 30). Le choix de travailler comme danseuse exotique l'isole, car elle est considérée par sa famille et ses connaissances en Guadeloupe comme une Marilisse. La Marilisse est l'un des deux stéréotypes sexuels issus de l'époque de l'esclavage qui ont été appliqués aux femmes noires - la Marilisse, introduite par Condé dans Heremakhonon ainsi que la châtaigne d'un proverbe créole : Fanm sé chatengn, nonm sé fouyapen (la femme est une châtaigne, l'homme est un fruit à pain). La Marilisse qui, selon Condé, a pris la figure de l'histoire était une esclave noire qui vivait avec un homme blanc et portait ses enfants (Entretien). Lorsque le stéréotype Marilisse est employé, il y a une accusation implicite selon laquelle la femme noire a tourné le dos à sa propre culture et choisi de vivre avec l'homme blanc. Condé sape le stéréotype de la Marilisse; elle révèle la réalité ambiguë et complexe à laquelle sont confrontées les femmes noires quand leur corps n'est pas considéré comme le leur. La Châtaigne, par opposition à la Marilisse, suggère la force des femmes des Caraïbes, mais implique également qu'avec cette force, elles devraient tolérer les abus et l'exploitation. Ces stéréotypes ont comme objectif de rabaisser les femmes noires des Caraïbes. Leur emploi confirme la persistance de l'héritage sexualisé de l'esclavage, et c'est lourd de conséquences pour les femmes noires. Pour Sahakian « By exaggerating Emma's bodily performance of the Marilisse and showing how the stereotype confusingly entraps her, Condé exposes how the memory of the damning seductress is ambiguously encoded in present-day French Caribbean women's bodies and behaviours » (Sahakian 398). Sahakian, en mettant l'accent sur le côté Marilisse du comportement d'Emma, ignore le deuxième rôle stéréotypé des femmes des Caraïbes, celui de la Châtaigne, la femme noire forte. Emma joue le rôle de Marilisse dans sa carrière, mais elle est néanmoins une femme noire forte qui a réussi à survivre seule. Dans une interview, Condé souligne que toutes les femmes noires des Caraïbes peuvent être non seulement belles, mais intelligentes : " They can be clever and talented, and wanted to use their brains. Most of the time they were seen as the exotic [...] Even when they are intelligent, the way most black women can succeed is with their bodies, through their figures. For me, Emma is the exemplification of the dilemma » (No Silence 545). Le dilemme auquel Emma est confrontée est qu'elle sent qu'elle n'aurait pas réussi à l'école de médecine, mais qu'elle est déterminée à réussir à sa guise. C'est clairement le comportement d'une Châtaigne têtue qui joue le rôle de la Marilisse pour obtenir ce qu'elle veut. Pourtant, le rôle de la Marilisse est un piège auquel elle n'échappera pas.

La relation d'Emma avec son rôle de danseuse exotique est complexe : elle est fière de son succès et de sa richesse, mais elle a également honte de la façon dont elle est perçue par le public. Elle est intelligente, bien informée et bien connectée, mais elle se sent tachée par son rôle. Emma projette son jugement de soi 
sur Ismaël et lui met des pensées dans la tête : " "Danseuse nue? - Oui, ma chère, elle montre son cul aux blancs!" »(Pension 22). Son emploi de danseuse exotique est déjà traumatisant en soi, reflet du traumatisme historique de l'esclavage. Emma emploie un vocabulaire associé à l'esclavage pour décrire l'amour et l'adoration qu'elle reçoit en tant que danseuse exotique : «Les hommes se sont courbés devant moi comme un champ de cannes à sucre sous la brise. Toutes les races. Toutes les couleurs » (Pension 13). La canne à sucre est clairement une allusion à l'esclavage. Ici, il y a un renversement des rôles; ce sont les hommes, en particulier blancs, qui sont soumis à une femme et une femme noire qui plus est. En outre, elles seraient mises sur un pied d'égalité selon cette image où tous les hommes se prosternent devant elle. De manière plus explicite, Ismaël utilise le langage de l'oppression pour décrire le comportement d'Emma envers lui. Selon Ismaël, la gentillesse d'Emma est un outil pour le soumettre : «Tout ce que tu veux, c'est me ligoter, m'asservir, me détruire avec ta gentillesse » (Pension 102). Ismaël utilise ce langage pour se distancier d'Emma, en suggérant qu'Emma le traite comme les victimes de l'esclavage ont été traitées. Emma est aussi une victime du traumatisme de la Traite. Comme le dirait Fanon, elle souffre d'une plaie laissée par la colonisation. À la fin de la pièce, il est évident que cette plaie l'a rendue folle. Emma est seule et à l'âge mûr; sa performance rituelle quotidienne pour un public blanc invisible reflète le traumatisme : «Je mets mes faux cils et le spectacle commence! » (Pension 127). Il y a une mise en abyme, car la pièce commence par un spectacle et se termine par un spectacle. Emma est une figure tragique. Le spectacle qu'elle interprète lui permet de revivre ses années de gloire en tant que danseuse exotique encore et encore; sa vie se répète éternellement. La présentation rituelle de son spectacle est une déclaration de son estime de soi et d'une identité dont elle veut être fière. Le portrait d'Emma à son apogée qui domine la scène est l'identité à laquelle elle s'accroche, une identité liée à la Marilisse.

Ni Emma ni Rosélie dans le roman Histoire de la femme cannibale n'apprécient pleinement l'impact caché de l'esclavage; pour Rosélie, ce sont ses traumatismes personnels qui la concernent. Rosélie est élevée par sa mère qui a été traumatisée par son mari infidèle et incapable de la nourrir. Les souvenirs d'enfance de Rosélie pourraient expliquer son incapacité à choisir de bons partenaires. Après avoir été larguée par son amant Salama Salama, un chanteur de reggae, Rosélie a été forcée de travailler comme prostituée. Sa période de prostituée était clairement traumatisante pour elle; elle utilise des euphémismes pour s'éloigner de cette période de sa vie : «Le plus vieux métier du monde, à ce qu'on prétend. Ce n'est pas de gaieté de cœur qu'une femme vend son corps » (Histoire 24). Que Stephen, celui qui devient son partenaire, l'ait sauvée de la prostitution a renforcé sa position dominante dans la relation. Alors que Rosélie écoutait son cœur quand elle a emménagé avec Stephen, elle n'a pas apprécié les conséquences de cette décision : «Rosélie, elle, avait les larmes aux yeux. Un sentiment de culpabilité la torturait qui ne devait plus la laisser en paix. On aurait dit que, de manière irréversible, elle avait coupé des liens dont elle ignorait elle-même la nature et la ténacité » (Histoire 37). Elle n'avait pas pensé au fait qu'en sortant avec Stephen, elle se coupait de son réseau de soutien d'amis noirs et de sa culture. De leur point de vue, elle jouait le rôle de la Marilisse, mais elle pourrait être considérée comme une Châtaigne, déterminée à survivre malgré, le racisme et le sexisme subtils et manifestes que toutes les femmes noires doivent affronter. Avec ses amis noirs, elle avait une identité, mais avec Stephen, elle n'en avait point, car elle n'était définie que par sa relation avec lui : «mon seul pays c'était Stephen » (Histoire 43).

Stephen a offert à Rosélie du réconfort et une certaine protection contre le monde pendant plusieurs années; elle s'est cachée du monde, cherchant refuge dans sa peinture. Rosélie était fascinée par l'horreur et tentait souvent de la représenter dans ses toiles. Sa peinture est thérapeutique, car dans son art, elle

Alternative francophone

https://journals.library.ualberta.ca/af/index.php/af 
exprime le traumatisme qu'elle ne peut exprimer autrement. Stephen ne comprend ni l'art ni les origines traumatisantes des toiles : " Pourquoi toujours des sujets si horribles? Corps démembrés, moignons, yeux crevés, rates, foies éclatés » (Histoire 63). Elle avait toujours aimé l'horreur, mais elle ne comprenait pas pourquoi. Stephen ne pouvait pas comprendre le lien entre les images horribles qu'elle peignait et l'héritage de l'esclavage et des traumatismes collectifs.

Malgré les images horribles que Rosélie peignait, elle n'avait pas accepté la mémoire collective comme une partie importante de son identité. Quand elle est interrogée sur la mémoire collective des traumatismes « le mal qu'ils nous ont fait» (Histoire 228) Rosélie répond : « Mon ami, je suis une égoïste. L'échec de mon présent m'absorbe plus que les blessures de notre passé » (Histoire 228). Elle évite de s'attacher aux traumatismes passés, mais sa relation avec le passé n'est aussi facile qu'elle aimerait faire croire, car sous la surface, une colère fait rage. Quand elle est en présence de Louw Jan, le mari raciste de son amie Sophie, son regard la ramène au temps de l'esclavage : « Debout derrière les sièges des maîtres, à agiter des éventails de plumes de paon pour chasser les mouches et rafraîchir leurs épaules en sueur. Couchée, jambes écartées, chair à plaisir du Maître. Dos courbé, lacéré par les coups de fouet de l'intendant » (Histoire 115). Il est évident que malgré tous ses efforts pour ignorer l'héritage de l'esclavage et le fait du racisme, elle ressent de la rage. Même lorsque Rosélie sortait avec Stephen, elle vivait dans la peur, s'identifiant aux victimes : «Ce qui l'avait effrayée, c'étaient les hommes. Blancs. Guides, gardiens, visiteurs autochtones, touristes étrangers. [...] Pas sa faute si elle souffrait du complexe des victimes et s'identifiant à ceux qui sont poursuivis » (Histoire 13-14). Stephen ne la protège pas du racisme; en fait, certains de ses comportements sont des exemples de racisme à peine voilé. Il défend les propos racistes de sa mère envers elle (Histoire 59-60). Même si Rosélie subit le racisme, elle s'éloigne toujours des discours de Césaire et de Fanon quand elle les entend (Histoire 66); c'est comme s'ils ne se rapportaient pas à elle. Après la mort de Stephen, elle doit faire face aux implications personnelles et collectives de sa marginalisation; elle est plus marginalisée dans son union avec Stephen qu'avant de le rencontrer. Elle n'a pas d'identité individuelle. Stephen, celui qui prétend la protéger, inflige un dernier traumatisme. Il lui cache son identité tout en refusant de reconnaître que c'est lui la plaie personnelle la plus profonde de sa vie. Rosélie est traumatisée à la fois par sa mort et la découverte que leur relation a été inauthentique; ce que Caruth appelle la réimposition de la plaie originelle. La notion de Caruth selon laquelle traumatisme se réimpose s'il n'est pas entièrement compris, peut s'appliquer à Histoire de la femme cannibale : «Derrière moi? C'est un cercle vicieux : si je n'ai pas compris, comment est-ce que je parviendrai, non à oublier, mais à me résigner? À repartir en cahotant dans la vie » (Histoire 337). Emma, en revanche, ne ressent pas la profondeur des sentiments de Rosélie; au fil des années, Emma a développé un mécanisme de défense, elle se réfugie dans son rôle sur scène.

Bien qu'Emma et Rosélie soient toutes deux victimes du traumatisme, Condé assigne à ses deux protagonistes le rôle de guérisseur d'autres personnes qui ont vécu le traumatisme. Elles s'identifient toutes deux comme guérisseuses. Alors qu'Emma a du mal à trouver l'amour elle-même, elle montre facilement de la compassion pour les hommes qui souffrent et qui ont besoin de guérison : «Tu ne sais pas combien d'hommes j'ai pris contre moi pour les guérir de leurs peurs. Ils arrivaient près de moi, noués, amers et frustrés. Et moi, il me suffisait de quelques mots, de quelques gestes » (Pension 79). Rosélie a également un don pour la guérison qu'elle a démontré à un jeune âge en aidant sa mère à s'endormir. Ayant désespérément besoin d'argent après la mort de Stephen, Rosélie crée une entreprise de guérison; elle soigne d'autres victimes de traumatismes, y compris Faustin qui n'arrive pas à dormir à cause des tourments et des tensions. Il devient son amant, mais il la quitte et alors qu'il l'invite à le rejoindre, Rosélie n'a pas assez de confiance en leur relation pour le suivre. Elle considère son nouvel emploi comme un moyen facile pour lui de rompre avec elle. La quête d'amour de Rosélie dans Histoire de la femme cannibale est compliquée. 


\section{LA QUETTE D'AMOUR}

Le traumatisme personnel et collectif que Rosélie et Emma ont vécu fait qu'il leur est difficile de faire partie d'une relation amoureuse, car de telles relations impliquent de la confiance. Pour Rosélie, chacune de ses relations se termine par le chagrin. Rosélie croit qu'elle partage un lien avec Stephen, mais après la mort de celui-ci, tout se révèle un mensonge. Le lien qu'elle partageait avec Stephen n'avait rien à voir avec l'amour. Toujours en ménage avec Stephen, elle a une liaison passionnée avec Ariel, un artiste qu'elle a rencontré à New York. C'est ce qui se rapproche le plus du vrai amour pour Rosélie, mais cette relation se termine quand Ariel est arrêté. Après avoir été libéré, Ariel tente de raviver l'amour, mais Rosélie choisit de rester avec Stephen. En tant que victime d'un traumatisme, elle passe d'une relation d'exploitation à une autre. À la fin du roman, après la mort de Stephen, Rosélie rejette une relation avec Manuel Desprez, un professeur de français et l'un des amis de Stephen : « Ce Manuel s'offrait à la consoler et de la trahison de Stephen et celle de Faustin. Mais, précisément, tous ces sauveurs providentiels ne la sauvaient pas. Ils ne faisaient que la détourner d'elle-même. Ils ne faisaient que la détourner de ce qui aurait dû être l'essentiel de ses préoccupations. Sa peinture » (Histoire 348). Elle brise le cycle de la dépendance; elle ne ressent plus le même besoin d'amour qui régnait sur sa vie pendant tant d'années.

Dans Pension les Alizés, Emma proclame toujours son besoin d'amour et elle tente désespérément de s'accrocher à Ismaël. Alors qu'Emma ne semble pas initialement intéressée par l'amour avec Ismaël, elle a soif de l'affection associée à l'amour pendant qu'ils ont des relations sexuelles : « Moi tout ce que je te demanderai, ce sera ta main sur mon épaule quand nous aurons fait l'amour et ton souffle sur mon cou [...] ton regard dans le mien parfois ton sourire. Une compréhension à demi-mot. » (Pension 57-58). Condé explore ici la dynamique interne d'une relation entre deux âmes endommagées où l'homme et la femme ont des attentes assez différentes. Vivant seul, il faut toujours être fort tandis que dans une relation, on peut se détendre un peu et baisser la garde; Emma se permet d'être vulnérable. Du point de vue d'Emma, tomber amoureux d'Ismaël l'a rendue faible : «J'étais bien, enfin presque, toute seule avec mes souvenirs, et puis tu es arrivé et je me suis mise à t'aimer...» (Pension 99). Du point de vue d'Ismaël, Emma est difficile à aimer, car en tant que femme noire, elle peut être intimidante : « Vous les négresses, vous ne savez pas aimer. Vous voulez qu'un homme soit fort, un mâle, au lit comme dehors du lit! (Pension 35). Ismaël a besoin d'une femme plus faible qu'il peut protéger. La relation d'Ismaël avec Emma est explicitement caractérisée par l'exploitation que celle de Stephen avec Rosélie. La relation est unilatérale; Ismaël tente de voler de l'argent à Emma et quand il est pris en flagrant délit, il s'insurge contre elle : « Je n'en veux plus de tes seins mous, de la peau de ton cou toute fripée, de ton ventre pareil à un plat de jello, et de ton sexe. De ton sexe surtout, insatiable » (Pension 94). Il s'agit d'une réaction vicieuse et viscérale de dégoût alors qu'Ismaël s'en prend à son apparence et en fait la pousse au sol. Malgré ces abus, le besoin d'amour d'Emma est si fort qu'elle l'oblige à dire qu'il ne voulait pas dire les choses horribles qu'il avait dites.

Plus tard, après leur réconciliation, Emma participe de manière ludique à un fantasme selon lequel elle accompagne Ismaël en Haïti. Le nom de la pièce vient de ce fantasme dans lequel Emma pourrait diriger un hôtel qui s'appellerait Pension les Alizés. Pour Emma, le fantasme dont Ismaël parle lui tient au cœur, car tout en étant fière d'être une danseuse exotique exceptionnellement bonne, elle a toujours honte de sa profession; un médecin noir à son bras lui donnerait la respectabilité tant souhaitée. Elle pourrait enfin se débarrasser de l'étiquette de Marilisse qu'elle a dû porter pendant tant d'années. Comme le note

Alternative francophone

https://journals.library.ualberta.ca/af/index.php/af 
Christiane Makward, alors que son nom évoque Emma Bovary, elle n'est pas aussi naïve que l'héroïne de Flaubert et elle se rend compte que la Pension les Alizés n'est qu'un rêve. Emma a l'habitude de jouer à la fantaisie, car elle revit chaque jour sa vie sur scène. Même si les attentes d'Emma sont plutôt modestes, sa quête d'amour n'est jamais satisfaite. Elle ne cherche pas un amour idéal, elle se contenterait de quelques petits signes d'affection. Elle a passé sa vie enlisée dans l'héritage sexualisé de la traite négrière, répondant aux fantasmes des hommes blancs et elle se retrouve maintenant seule. Sans autre identité que celle d'une danseuse exotique noire, elle retrouve difficilement un minimum d'amour.

\section{LA QUÊTE D'IDENTITÉ}

Emma et Rosélie ont toutes deux cherché leur identité tout au long de leur vie. L'identité d'Emma est d'abord menacée lorsqu'elle quitte la faculté de médecine. Elle commence à travailler comme danseuse exotique et on veut qu'elle change de nom : «"Emma Boisgris, cela fait trop, trop ordinaire” J'ai refusé. Emma Boisgris, j'étais née. Emma Boisgris, je resterais » (Pension 13). Le nom est un élément fondamental de son identité. Bien qu'Emma refuse de compromettre cette partie de son identité, ses quêtes identitaire et amoureuse sont compliquées par le traumatisme historique de la traite négrière : «The dramaturge exposes the theme of Creolization through representations of their conditions of migration, sense of loss in the city of Paris, and troubled identity. Their encounter enables them to uncover postplantationary remnants that paralyze their relationship and stunt their identity formation » (Francis 42). Il est évident dès la première scène de la pièce qu'elle participe à l'héritage sexualisé de l'esclavage et que c'est ainsi que les autres la verront : «La nouvelle Joséphine Baker, c'est comme cela qu'ils m'appelaient! Mais moi, je n'aimais pas qu'ils m'appellent ainsi. Quand je voyais cela dans leurs journaux, je n'étais pas contente. Parce que je n'ai jamais accepté de ceinture de bananes, moi » (Pension 11). Joséphine Baker était une danseuse exotique afro-américaine qui jouait à Paris. La ceinture de bananes est un symbole exotique, sexuel et raciste qui lui est associé; elle la portait lors de ses performances. Bien que Joséphine Baker ne fût pas une esclave, elle était complice de la représentation de l'héritage de l'esclavage ayant accepté de jouer pour un public essentiellement blanc (mais elle fut aussi une « châtaigne »). Emma rejette cette association avec Joséphine Baker, reflet de sa relation complexe avec sa carrière de danseuse exotique; elle est fière de sa vie, mais aussi gênée : « Peut-être quand moi aussi je mourrai, ils cesseront de chuchoter : "Danseuse nue, danseuse nue" pour se mettre à parler de mes qualités! » (Pension 85). Elle se sent jugée pour sa profession. Alors qu’Emma aurait aimé retourner en Guadeloupe, le retour est rendu plus difficile par la profession choisie : « in making a spectacle of her "exotic and fiery" créolité, Emma actually widened the gulf between her and her native island. She could no longer return home, since her parents and community condemned her chosen profession » (Beach 199). Emma est coupée de ses racines par sa profession, ce qui rend plus difficile l'établissement de son identité.

L'identité d'Emma est profondément liée à son apparence. Elle est consciente du fait qu'elle n'est plus aussi jolie qu'elle ne l'était : «Qu'est-ce que vous auriez dit si vous m'aviez vue à vingt ans » (Pension 37). Son apparence est importante pour elle : « Heureusement, je n'ai pas grossi. Mon corps est svelte » (Pension 86). En tant que danseuse exotique noire, elle est marginalisée de trois manières : en tant que noire, en tant que femme et en tant que danseuse exotique. Elle s'identifie toujours à ce rôle et elle est fière de ses spectacles, elle les répète comme l'affirmation d'une identité. Elle ne s'est jamais mariée, mais était manifestement la concubine de riches hommes blancs qui l'ont récompensée. Elle n'est pas démunie dans sa vieillesse, mais solitaire comme le montre sa relation avortée avec Ismaël. Elle est considérée comme une Marilisse par les Guadeloupéens, mais elle conserve toujours sa dignité en s'identifiant à une version plus jeune d'elle-même lorsqu'elle était indépendante et dans la fleur de l'âge. 
Pendant la plus grande partie de sa vie, Rosélie n'a jamais eu de sentiment d'identité indépendante. Son identité était façonnée selon la vie des hommes et les suivait où qu'ils aillent. Elle passait en somnambule à travers la vie; c'est la mort de Stephen qui la réveille. C'est un grand choc quand Dido, l'amie de Rosélie, révèle ses sentiments véritables envers Stephen : «Non, je ne l'aimais pas. C’était un égoïste, un despote. Il t'empêchait d'être toi-même » (Histoire 176). Comme pour confirmer l'opinion de Dido, Rosélie remet en question sa propre identité : « Mais qui suis-je? Quelle bête, quel poisson carnivore? » (Histoire 176). Rosélie avait refusé d'affronter le problème de son identité jusqu'à ce qu'il lui soit imposé avec la mort de Stephen. Elle se rend finalement compte que Stephen l'a utilisée comme écran pour cacher sa propre identité d'homosexuel. Pendant de nombreuses années, elle fait partie d'une relation inauthentique sans réfléchir à sa propre identité.

Elle commence à comprendre sa véritable identité lorsqu'elle se voit dans les yeux de Fiéla, une femme accusée de meurtre dont elle a vu la photo dans un journal. Fiéla est accusée d'avoir tué son mari blanc et d'avoir mis son corps démembré dans le congélateur. Les médias appellent Fiéla la femme cannibale qui donne le titre au roman. Fiéla émerge comme un modèle important pour Rosélie qui s'identifie d'abord à Fiéla par leur ressemblance physique. Quand Rosélie voit son image, elle pense : «Elle a mon âge. Elle n'est pas belle. Elle pourrait être moi » (Histoire 96). Cette identification avec Fiéla, chargée d'émotion, change sa vie.

Rosélie's connection with Fiéla reveals the myth of her relationship with Stephen, releasing the identity that she believed emanated from him and allowing her to take possession of it for herself. Through her relationship with Fiéla, la femme cannibale Rosélie transcends Stephen's domination and the cannibalism of which she was a victim is sublimated into a communion of identity with Fiéla. (Barnard 332)

Debbie Barnard suggère en outre que l'identité de Rosélie est restaurée grâce à son identification à Fiéla : «Rosélie's identity has been devoured, or cannabalized by her relationship with Stephen; it is through her identification with Fiéla, the femme cannibale that it is restored » (Barnard 328). Cependant, il est évident que Rosélie n'avait pas d'identité indépendante des hommes de sa vie et ce n'est qu'en se reconnaissant dans Fiéla qu'elle se libère enfin de l'héritage de l'esclavage et est en mesure d'établir sa propre identité. Au cœur de l'identification de Rosélie avec Fiéla se trouve leur expérience commune du racisme en tant que femmes noires. En fin de compte, Fiéla l'inspire à devenir forte. Son identification avec Fiéla établit une nouvelle relation avec la mémoire collective et lui permet de se forger une nouvelle identité. Elle refuse de se définir par la mémoire collective traumatisante associée à la traite des esclaves. Elle s’identifie avec Fiéla, un personnage qui n'est pas/plus cannibalisée, mais qui cannibalise.

Emma et Rosélie ont recours à l'expression artistique comme moyen d'établir leur identité, Rosélie à travers la peinture et Emma à travers la danse exotique. Les toiles de Rosélie ont souvent canalisé les horreurs infligées par la traite négrière. En regardant le visage de Fiéla, Rosélie voit les traumatismes que les femmes noires continuent de subir. Inspirée par Fiela, elle peint une toile qu'elle appelle femme cannibale, nom qui suggère une caractérisation. L'art de Rosélie lui permet de développer une identité indépendante tandis que l'art d'Emma s'articule autour de son apparence et de la danse exotique. L'identité d'Emma n'aboutit à rien, car son apparence se détériorera inévitablement avec le temps, tandis que celle de Rosélie se développe : «A travers l'art, Rosélie se redéfinit et renaît en femme non seulement artiste mais aussi cannibal, ne se laissant plus dévorer par le monde mais le dévorant à son tour

Alternative francophone

https://journals.library.ualberta.ca/af/index.php/af 
pour mieux le recréer à sa mesure » (Migraine-George 507). L'identification de Rosélie avec Fiéla l'a revigorée et lui a donné un sentiment d'identité et de but.

\section{CONCLUSION}

Près de vingt ans après avoir écrit la pièce Pension les Alizés, Maryse Condé revient sur un sujet similaire, la création de l'identité et la recherche de l'amour dans le contexte de l'héritage sexualisé de l'esclavage et du traumatisme associé avec l'écriture du roman Histoire de la femme cannibale. Condé manipule les rôles stéréotypés de la Marilisse et du Châtaigne dans les deux œuvres dans un terrain complexe et changeant. Le retour de Condé sur le même sujet, présenté d'abord dans Pension les Alizés, lui permet de présenter Rosélie d'une manière plus complexe que ce qui était possible dans le texte court d'une pièce; cela permet aussi au lecteur de voir l'évolution de ses idées. Emma et Rosélie affrontent les mêmes problèmes pour créer une identité et trouver l'amour tout en traitant des traumatismes personnels et collectifs. Dans Pension les Alizés Emma est incapable de créer une identité au-delà du rôle de Marilisse et de danseuse exotique; alors qu'elle désire l'amour, elle n'a jamais pu trouver un homme qui traverserait la vie avec elle. Dans Histoire de la femme cannibale, Rosélie, malgré ses relations multiples, y compris celles de longue durée, n'a pas non plus de succès à trouver un grand amour. Cependant, la pensée de Condé ici semble avoir évoluée, car elle est plus optimiste pour Rosélie qui est représentée comme un personnage ayant connu seulement un progrès limité. Au départ, Roselie pouvait être considérée comme une Marilisse, car elle était dominée par Stephen, un homme blanc. Inspirée par Fiéla, Rosélie se libère de l'héritage sexualisé de l'esclavage. Sa ténacité qui a toujours été là, est révélée à la fin du roman. Devant l'offre d'amour de Manuel, Rosélie, contrairement à Emma qui poursuit Ismaël jusqu'à ce que ce ne soit plus une option, choisit plutôt son art. Condé ne voyait aucun espoir pour Emma dans Pension les Alizés. Malheureusement, elle est toujours contrôlée par l'héritage sexualisé de l'esclavage, car chaque jour, elle répète les mêmes scènes pour son public invisible d'hommes blancs. Dans Histoire de la femme cannibale, Condé montre qu'une identité en dehors de l'héritage sexualisé de l'esclavage peut se former par ceux qui ont été traumatisés par l'héritage de l'esclavage. Grâce à son identité, Rosélie peut désormais être dans une relation avec un homme sur un pied d'égalité si elle le souhaite. En affrontant les traumatismes de sa vie, Rosélie semble retrouver sa voix/voie. 


\section{BIBLIOGRAPHIE}

Barnard, Debbie. «Serving the Master: Cannibalism and Transoceanic Representations of Cultural Identity. » International Journal of Francophone Studies, vol. 8, no. 3, 2005, pp. 321-339.

Beach, Cecilia. « The Mother(land) in Plays by Maryse Condé. » The Mother in/and French Literature, Editions Rodophi Brill Academic Publishing, 2000, pp.195-208.

Caruth, Cathy. Unclaimed Experience: Trauma, Narrative, and History. Johns Hopkins University Press, 2016.

Condé, Maryse. En attendant le bonheur (Heremakhonon) : roman. Seghers, 1988.

Condé, Maryse, et Françoise Pfaff. Entretiens avec Maryse Condé : suivis d'une bibliographie complète. Editions Karthala, Paris, 1993.

Condé, Maryse. Histoire de la femme cannibale : roman. Folio, 2003.

Condé, Maryse. Pension les Alizés. Mercure de France, 1988.

Francis, Gladys M. Odious Caribbean Women and the Palpable Aesthetics of Transgression. Lexington Books, an imprint of The Rowman \& Littlefield Publishing Group, 2017.

Fanon, Frantz. Les Damnés de la Terre. Maspero, 1968.

Halbwachs, Maurice, Gérard Namer, et Marie Jaisson. La mémoire collective. A. Michel, 1997.

Lewis, Barbara, et Maryse Condé. « No Silence: An Interview with Maryse Condé. » Callaloo, vol. 18, no. 3, Johns Hopkins University Press, 1995, pp.543-550.

Makward, Christiane P. « Reading Maryse Conde's Theatre. » Callaloo, Johns Hopkins University Press, vol. 18, no. 3, 1995, pp.681-689.

Migraine-George, Thérèse. «De Traversée de la mangrove à Histoire de la femme cannibale : 1'art comme arme miraculeuse chez Maryse Condé. » Romanic Review, vol. 101, no. 3, 2010, pp. 497519.

Sahakian, Emily. « Beyond the Marilisse and the Chestnut: Shattering Slavery's Sexual Stereotypes in the Drama of Ina Césaire and Maryse Condé. » Modern Drama, vol. 57, no. 3, 2014, pp.385-408. 